\title{
Declining Foraging Efficiency in the Middle Tennessee River Valley Prior to Initial Domestication - ERRATUM
}

Elic M. Weitzel

DOI: 10.1017/aaq.2018.86, Published by Cambridge University Press, 1 April 2019

The original publication of this article (Weitzel 2019) included an incorrect Spanish abstract. The article has since been corrected.

The publisher apologizes for the error.

\section{Reference Cited}

Weitzel, Elic M.

2019 Declining Foraging Efficiency in the Middle Tennessee River Valley Prior to Initial Domestication. American Antiquity 84:191-214. 